\title{
Real-time Monitoring of Multiphase Flow within Rock Miniplugs using 2D X-ray Imaging
}

\author{
Vera Pletneva $^{1}$, Dmitry Korobkov ${ }^{1}$, Ivan Yakimchuk ${ }^{1}$ \\ ${ }^{1}$ Schlumberger, Schlumberger Moscow Research (SMR) Center \\ 119285, Pudovkina str. 13, Moscow, Russia \\ vpletneva@slb.com; dkorobkov@slb.com; iyakimchuk@slb.com
}

\section{Extended Abstract}

One of the contemporary concepts widely applied to rock analysis nowadays includes construction of digital models followed by the subsequent numerical simulations at pore scale of various physical processes taking place under real conditions [1-2]. In the framework of this concept the rock permeability is determined using single- or multiphase fluid flow simulations on X-ray microtomography (X-ray microCT) image-based digital model of the sample. X-ray microCT is a nondestructive experimental technique, which discloses inner structure of the rock [3]. One of the most significant concern in 3D microCT imaging is real-time monitoring that allows one to observe the internal processes in dynamics [4]. For specific cases some progress can be found in literature [5]. To address this time resolution challenge one can utilize simpler and faster 2D X-ray imaging. Even in 2D mode real-time imaging can be valuable, e.g. for validation and tuning of digital rock models. We are focused on monitoring of saturation profiles during multiphase flow through a rock miniplug sample $(8 \mathrm{~mm}$ in diameter). Standard methods, such as mass balance, face problems related to small sample size and very low mass values to be controlled. Developed approach allows monitoring of fluid distribution and fluid content estimation inside the core at different time using laboratory table-top SkyScan 1172 X-ray microtomograph (Bruker MicroCT: Kontich, Belgium) as the base X-ray imaging system. The approach includes the development of hardware solution (special core holder for fluid filtration and fluid injection system with working fluids, tubings and ohmmeter terminals connections inside limited volume of microCT), which is capable to work at conditions similar to reservoir (up to $40 \mathrm{MPa}$ ), complimented by image processing and interpretation procedure.

At the moment, filtration experiments have been successfully carried out on several sandstone miniplugs of $15-25 \%$ porosity. Mixture of $20 \mathrm{wt} . \%$ of potassium iodide (KI) in distilled water was used as the water (brine) phase, while n-decane was used as the oil phase. The selection of the fluids was driven by a trade-off between the aim of replicating a reservoir system whilst having enough relative contrast to distinguish the phases in X-ray images.

2D images obtained using microCT setup represent the spatial distribution of the intensity of X-ray beam passed through the PEEK cell with the sample inside. Over the course of the experiment, intensity values for the constant parts of the image (cell components) significantly varied. This observation suggests the existence of variations in X-ray tube radiation with time. To compensate for this effect, we developed a normalization procedure. The distributions of fluid saturation in the core were obtained. One of the main outputs of fluid filtration experiments is the information about fluid front evolution during different injection stages. We used values for the average intensity along each of the image rows to represent characteristic saturation profile of brine/oil phases. The methodology was verified using conventional method of indirect sample saturation estimation (by electrical resistance measurements).

\section{References}

[1] D. Klemin, A. Nadeev and M. Ziauddin, "Digital rock technology for quantitative prediction of acid stimulation efficiency in carbonates" in Proceedings of SPE Annual Technical Conference and Exhibition, Houston, TX, USA, 2015, SPE-174807-MS.

[2] O. Y. Dinariev and N. V. Evseev, "Modeling of nanoscale liquid mixture transport by density functional hydrodynamics," Phys. Rev. E., vol. 95, no. 6, p. 063307, 2017.

[3] T. M. Buzug and D. N. Mihailidis, "Computed tomography: from photon statistics to modern cone-beam CT," Med. Phys., vol. 36, no. 8, p. 3858, 2009. 
[4] S. Berg, R. Armstrong, H. Ott, A. Georgiadis, S.A. Klapp, A. Schwing, R. Neiteler, N. Brussee, A. Makurat, L. Leu, F. Enzmann, J.-O. Schwarz, M. Wolf, F. Khan, M. Kersten, S. Irvine and M. Stampanoni, "Multiphase flow in porous rock imaged under dynamic flow conditions with fast X-ray computed microtomography," Petrophysics, vol. 55, no. 4, p. 304, 2014.

[5] M. Andrew, H. Menke, M. J. Blunt and B. Bijeljic, "The imaging of dynamic multiphase fluid flow using synchrotronbased X-ray microtomography at reservoir conditions," Transport Porous Med., vol. 110, no. 1, p. 1, 2015. 